\title{
Convolutional Neural Networks for Brain Tumor Segmentation Using Different Sets of MRI Sequences
}

\author{
Masoomeh Rahimpour, Karolien Goffin, Michel Koole
}

\begin{abstract}
In clinical practice, multi-sequence MRI protocols for brain tumor segmentation are not standardized and therefore a flexible segmentation approach is needed which makes optimal use of all available MRI data. In this study, we present and evaluate an early and late fusion Convolutional Neural Network (CNN) based on DeepMedic architecture to segment brain tumor using different combinations of multi-sequence MRI datasets. While for the early fusion approach, we trained a dedicated CNN for all possible combinations of MRI sequences, the late fusion approach is a more generic approach where we trained an independent $\mathrm{CNN}$ for each type of MRI sequence and merged the feature maps using a fully connected layer to generate the final segmentation. Compared to an early fusion $\mathrm{CNN}$, the segmentation performance of the late fusion approach was very similar while it provides more flexibility in terms of combining all available MRI data.
\end{abstract}

Index Terms-Brain tumor segmentation, Multi-sequence MRI, Deep learning.

\section{INTRODUCTION}

C URRENT developments for the MRI-based segmentation of brain tumors are mainly based a standardized, fixed number MRI sequences. For instance, for the Brain Tumor Segmentation challenge (BRATS), training and evaluation datasets include $T_{1}, T_{1 c e}, T_{2}$ and $T_{2}$-FLAIR MRI data to allow a voxelwise classification between healthy and abnormal brain tissue. Therefore, most of the recent studies on brain tumor segmentation use a deep learning framework where all available MRI sequences are merged and fed into the network for classification purposes. This early fusion strategy takes into account all information from multi-sequence MRI datasets early in the training process and trains a dedicated network for a specific set of MRI sequences. However, in clinical practice, multi-sequence MRI protocols for brain tumor imaging are rarely standardized, such that these four specific MRI dataset are not always available for every patient or part of these datasets is acquired suboptimally at a lower resolution. As an example, the frequency of different MRI sequences

\footnotetext{
Manuscript received December 21, 2019. This project has received funding from the European Union's Horizon 2020 research and innovation programme under the Marie Sklodowska-Curie grant agreement No 764458.

Masoomeh Rahimpour is with the Department of Imaging and Pathology, Nuclear Medicine \& Molecular Imaging, KU Leuven, Belgium (e-mail: masoomeh.rahimpour@kuleuven.be).

Karolien Goffin is with the Department of Imaging and Pathology, Nuclear Medicine \& Molecular Imaging, KU Leuven, Belgium (e-mail: karolien.goffin@kuleuven.be).

Michel Koole is with the Department of Imaging and Pathology, Nuclear Medicine \& Molecular Imaging, KU Leuven, Belgium (e-mail: michel.koole@kuleuven.be).
}

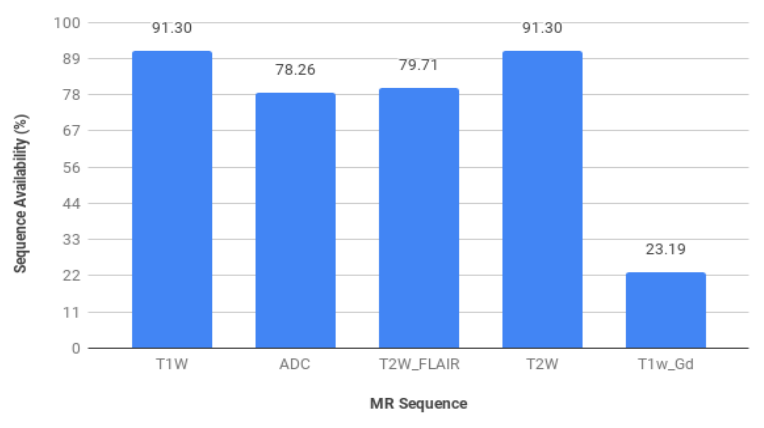

Fig. 1. MR modality distribution in UZ Leuven patients with glioma in 2017.

acquired at UZ Leuven in 2017 for the treatment and followup of glioma patients is presented in Fig. 1. These patients were referred to the department of Nuclear Medicine for a ${ }^{18} \mathrm{~F}$-fluoroethyl-L-tyrosine $\left({ }^{18} \mathrm{~F}-\mathrm{FET}\right)$ PET scan. These data indicate that $T_{1}$ and $T_{2}$ weighted MR images are the most widely available sequences while $T_{1 c e}$ MR sequence is not performed on a regular basis. In order to achieve optimal performance for all routine clinical datasets, a segmentation algorithm needs to take into account the availability of different MRI sequences. Therefore, we considered two CNN approaches for brain tumor segmentation using different sets of MRI sequences. As a first approach, we trained and evaluated a dedicated, early fusion CNN for each combination of MRI sequences. However, for this approach, training datasets are limited by the MRI sequence with smallest dataset. Therefore, we also considered a late fusion approach where a network is trained separately for each available MRI sequence and then a combination of labels is considered as the final labeling result. Compared to early fusion where all available input datasets are concatenated in low-level feature space and some specific information captured by single type of input dataset, a late fusion approach allows to focus more on the individual strength of every MR sequence and to better handle of training the network in presence of different sets of MR sequence.

\section{Materials And Methods}

We evaluated the segmentation approach using the data provided by BRATS2017 challenge. This dataset consists of 210 cases with high grade glioma (HGG) and 75 cases with low grade glioma (LGG) scans. From this dataset, $80 \%$ of 


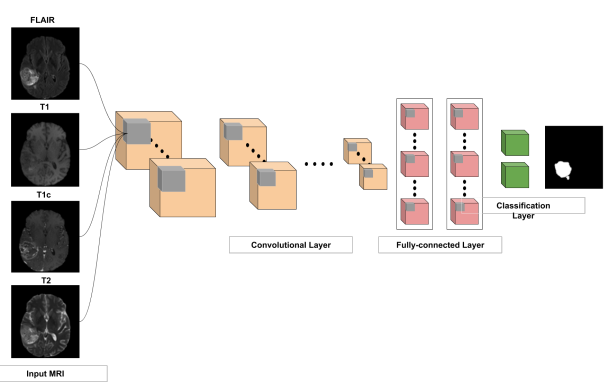

(a)

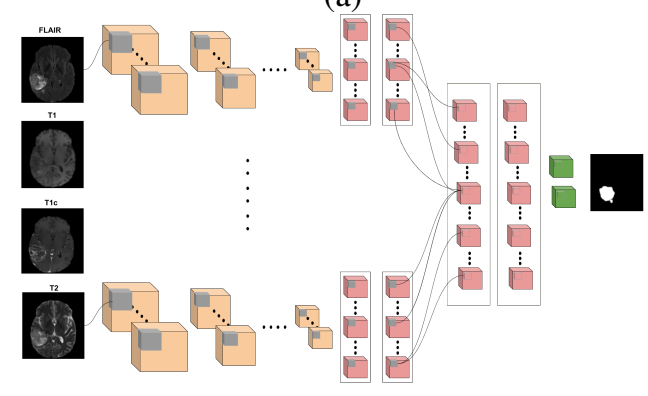

(b)

Fig. 2. Comparative schematic of (a) Early fusion and (b) Late fusion.

the dataset are selected randomly for training, $10 \%$ for validation and $10 \%$ for testing. Our segmentation approach follows DeepMedic-based CNN [1] architecture which performs on $3 \mathrm{D}$ volumes and the training procedure is performed in a patch-wise fashion. DeepMedic consists of multiple pathways allowing to extract the features from multiple resolutions independently. This multi-scale processing enables the network to achieve a large receptive field for the final classification while keeping the computational cost low.

Since the relative distribution of tumor and healthy tissue voxels in brain images is unbalanced, we have an inherent class imbalance problem in our dataset. To deal with this issue, we applied the patch sampling procedure in which $50 \%$ of batches consist of patches centered on healthy tissue voxels and the other $50 \%$ located on the tumor tissue voxels. Furthermore, we explored the performance of different loss functions including dice, weighted cross entropy and focal losses in dealing with class-imbalance [2].

In early fusion approach, we had a unified CNN being fed by image batches consisting of multiple MRI sequences of a patient, while in late fusion, we trained a network which consists of four independent CNNs for every individual modality and then merges the high-level extracted features before feeding them to the final fully-connected layers to output the final segmentation. A comparative schematic for early fusion and late fusion implementation has been shown in Fig. 2. The CNNs for processing every individual modality follows the same architecture. Every MR sequence has a different contribution to the final segmentation. This is why we also explored the performance of the network trained on every individual MR sequence.

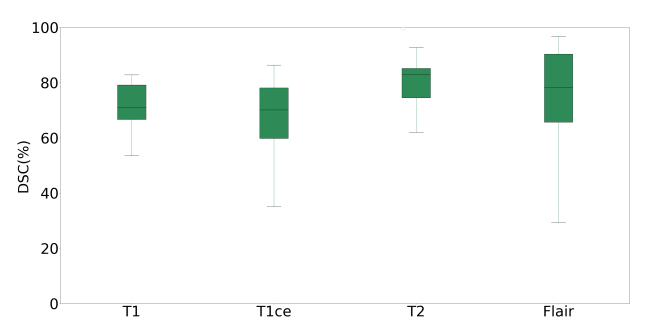

Fig. 3. Average DSC achieved by the network trained on every single MR modality

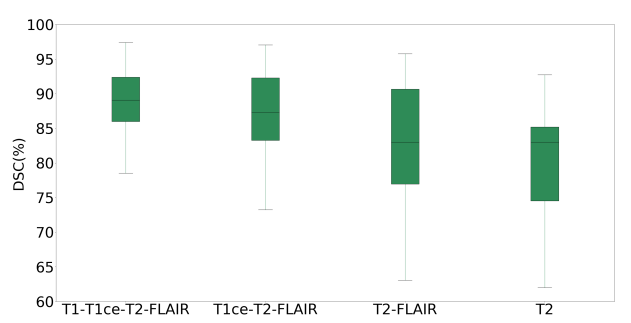

Fig. 4. Average DSC achieved by the network trained on most consistent MR modality combinations.

TABLE I

PERFORMANCE OF THE NETWORK ON TEST DATASET USING DIFFERENT OBJECTIVE FUNCTIONS.

\begin{tabular}{lccc}
\hline \hline Loss function & DSC & Precision & Sensitivity \\
\hline \hline Weighted Cross Entropy & 88.33 & 91.44 & 87.56 \\
\hline Dice & 87.21 & 90.44 & 85.83 \\
\hline Focal & 85.28 & 90.57 & 84.40 \\
\hline \hline
\end{tabular}

TABLE II

A COMPARISON OF SEGMENTATION RESULTS OBTAINED BY EARLY FUSION AND LATE FUSION.

\begin{tabular}{lcc} 
Modality Permutation & Early Fusion & Late Fusion \\
\hline \hline$T_{1}, T_{1 c e}, T_{2}$, Flair & 88.33 & 89.35 \\
\hline$T_{1}, T_{2}$, Flair & 84.32 & 85.86 \\
\hline$T_{1 c e}, T_{2}$, Flair & 86.50 & 85.89 \\
\hline$T_{2}$, Flair & 82.66 & 86.04 \\
\hline \hline
\end{tabular}

\section{RESULTS}

The implemented CNN is a dual-pathway network with each pathway consisting of 11 layers including 8 convolutional, 2 fully connected, and 1 classification layer. Table I, represents the performance of this segmentation framework on three different loss functions to explore their efficiency in dealing with imbalanced dataset. The average dice score (DSC) achieved by the network trained on individual MR sequences is shown in Fig. 3. Figure 4 depicts the average DSC achieved by the early fusion network trained on most performant combinations of MR sequences. Table II, compares the segmentation results obtained by late fusion and early fusion network.

\section{Discussion AND SUMmary}

For the early fusion approach, feature maps are concatenated in low-level feature space such that the network can only con- 
sider a simple relationship between different MRI sequences. On the other hand, the late fusion approach combines the feature maps extracted for every type of sequence in the high-level layer such that it can make better use of specific information of each type of sequence. While segmentation performance of both approaches is very similar, the late fusion approach is more generic and provides more flexibility in terms of combining all available MRI data. In the next step, we will further improve the late fusion implementation by applying a joint representation learning based on feature maps obtained by sequence-specific networks.

\section{REFERENCES}

[1] K. Kamnitsas, C. Ledig, V. F. Newcombe, J. P. Simpson, A. D. Kane, D. K. Menon, D. Rueckert, and B. Glocker, "Efficient multi-scale 3d cnn with fully connected crf for accurate brain lesion segmentation," Medical image analysis, vol. 36, pp. 61-78, 2017.

[2] T.-Y. Lin, P. Goyal, R. Girshick, K. He, and P. Dollár, "Focal loss for dense object detection," in Proceedings of the IEEE international conference on computer vision, 2017, pp. 2980-2988. 